\title{
Polarized neutron diffraction on a diamagnetic bismuth single crystal
}

\author{
K. Prokeš \\ Helmholtz-Zentrum Berlin für Materialien und Energie, Hahn-Meitner Platz 1, 14109 \\ Berlin, Germany \\ F. Mezei
}

European Spallation Source ESS AB, Box 176, 22100 Lund, Sweden

A. Gukasov

Laboratoire Léon Brillouin, CEA-Saclay, 91191 Gif sur Yvette Cedex, France

\begin{abstract}
A large single crystal of bismuth has been studied between $2 \mathrm{~K}$ and $150 \mathrm{~K}$ by means of polarized neutron diffraction in fields up $6.2 \mathrm{~T}$ applied along the trigonal axis. The recorder flipping ratios are very small in agreement with the diamagnetic response of bismuth. Maximum entropy reconstruction shows that the leading contribution to the magnetic field induced magnetization is indeed associated with electron states centered at bismuth. However, small contribution stems from states placed outside bismuth atoms.
\end{abstract}

Keywords: Bi single crystal, diamagnetism, polarized neutron diffraction 


\title{
Polarized neutron diffraction on a diamagnetic bismuth single crystal
}

\author{
K. Prokeš \\ Helmholtz-Zentrum Berlin für Materialien und Energie, Hahn-Meitner Platz 1, 14109 \\ Berlin, Germany \\ F. Mezei
}

European Spallation Source ESS AB, Box 176, 22100 Lund, Sweden

A. Gukasov

Laboratoire Léon Brillouin, CEA-Saclay, 91191 Gif sur Yvette Cedex, France

\section{Introduction}

Bismuth is at ambient pressure diamagnetic. The diamagnetism that is connected with changes in electron trajectories induced by applied magnetic field exists in all the materials. However, in majority of the cases it is much smaller with respect to other magnetic contributions. Nevertheless, several materials that include graphite and bismuth exhibit anomalously large diamagnetism $[1,3,2]$. Bismuth is reported to be anisotropic with the $c$-axis magnetic susceptibility being in absolute value approximately $30 \%$ smaller than with field directed perpendicular to it [3].

At low temperatures is the diamagnetic response to magnetic field in bismuth oscillating due to crossing of Landau and Fermi levels, phenomenon known as the de Haas - van Alphen effect [4]. Diamagnetism in bismuth has been shown that it originates from inter-band features of a specific band structure with a small, temperature dependent, energy gap $[2,5,6,7,8,9]$. Similar effect has been found to be responsible for anomalous diamagnetism in graphene and other low-dimensional semiconductors [10].

Bismuth's crystal structure (space group R $\overline{3} 2 / \mathrm{m}$, no. 166) can be described in three different ways using either rhomboedral or hexagonal unit cells. In the present work we use, when addressing Bragg reflection indexes, 
the hexagonal notation with lattice parameters $\mathrm{a} \approx 4.5 \AA$ and $\mathrm{c} \approx 11.8 \AA$. Within this lattice, $\mathrm{Bi}$ atoms occupy the $6 \mathrm{c}(0,0, \mathrm{z})$ site with $\mathrm{z}$ position parameter of about 0.23 . Bismuth is a semi-metal with extremely small Fermi surface, consisting from a hole pocket aligned along the trigonal axis and three Dirac electron ellipsoidal pockets that are tilted by about 6 degrees out of the bisectrix - binary plane. [11] The low concentration of itinerant electrons leads to confinement of electrons to the lowest Landau level already at rather low fields [12]. Various very interesting phenomena have been studied in bismuth. For instance, the electrical resistivity increases at low temperatures with application of a moderate magnetic field by several orders of magnitude with respect to its zero field value $[13,14]$ and Nernst, Seebeck and Hall effects show oscillatory dependencies [16, 17, 18, 19, 20, 21, 15]. Oscillations have been detected even in ultrasound measurements [22].

Our motivation for this work was to experimentally verify whether it is possible to determine the magnetization distribution in bismuth and compare it with the magnetic susceptibility measurements. In this work we compare experimental data obtained at two different fields, close to the maximum and minimum of the magnetization oscillations.

It is well known that the use of polarized neutron diffraction (PND) experiment enhance the sensitivity to magnetism with respect to the unpolarized neutron diffraction experiment enormously. [24] The PND technique has been frequently used in determinations of the electron redistribution caused by the applied magnetic field near the Fermi surface. The magnitude of the signal depends on the density of states and is capable to give the direct information on the distribution of the magnetization in the unit cell and allows for the identification of different contributions to the magnetic moments [23]. In a pioneering work of C.G. Shull and R.P. Ferrier [24] it has been demonstrated that it is possible to discriminate by means of this method between the nuclear and electronic contributions to the scattering intensity and by comparing the intensities to a calculated magnetic form factors to disclose the electronic configuration. Later on, Stassis argued that PND experiment should be sensitive enough to separate the magnetic contribution due to diamagnetic current induced by applied magnetic field [25].

\section{Experimental}

A $2.5 \mathrm{~cm}^{3}$ large bismuth single crystal of $99.999 \%$ purity has been prepared on a by Bridgeman method by MaTeck GmbH. The X-ray and neutron 
Laue technique has shown that it consists from a majority grain that comprises $90 \%$ of the sample volume and $10 \%$ volume grain about 0.7 degrees away. Such a quality (although not perfect) does not hamper main conclusions of this work. The crystal has been glued to an aluminium rod (used later to attach the crystal in the course of the diffraction experiment inside the vertical field cryomagnet) with its hexagonal axis approximately vertical.

Part of the single crystal has been cut using a diamond wire and investigated by means of magnetic bulk measurements. Magnetization (M) curves between 2 and $100 \mathrm{~K}$ were measured in fields up to $14 \mathrm{~T}$ in a Physical Property Measurement System apparatus. The dc magnetic susceptibility $\chi=$ $\mathrm{M} / \mu_{0} H$, where $\mu_{0} H$ denotes the applied magnetic field was measured in the temperature range $2-300 \mathrm{~K}$ in magnetic fields up to $10 \mathrm{~T}$.

To determine the magnetic structure factors, we have utilized the polarized neutron diffraction (PND) technique. PND experiment has been carried out on 5C1 diffractometer [26] installed at the ORPHÉE 14 MW reactor of the Léon Brillouin Laboratory, CEA/CNRS Saclay. Neutrons at this instrument are from the source monochromated to a wavelength $\lambda=0.84 \AA$ and polarized by $\mathrm{Cu}_{2} \mathrm{MnAl}$ Heusler crystal. The polarization of the beam is $90 \%$. An adiabatic radio-frequency flipper is installed between the polarizer and superconducting magnet capable to produce vertical fields up to $6.2 \mathrm{~T}$. A great advantage of this instrument is a large position-sensitive detector that covers a large portion of the reciprocal space above and below the scattering plane. Data were collected by rotating the crystal around its vertical axis (parallel to the applied field) with a step of $0.2 \mathrm{deg}$ with collection time of 20 s per point. However, since Bragg reflections are broader and the reflection condition is for a certain reflection satisfied for a range of rotation angles, the real collection time was typically about $120 \mathrm{~s}$. The sample has been rotated by $270 \mathrm{deg}$. In this way, symmetry equivalent Bragg reflections were covered.

According to the refined orientation matrix obtained from all detected reflections using in-house developed software, the magnetic field has been applied 3.5 degrees from the hexagonal $c$-axis. In total, we have collected at $2 \mathrm{~K}$ and $6.2 \mathrm{~T} 87$ inequivalent Bragg $(h k l)$ reflections (identical number of reflections were collected also with field of $4.5 \mathrm{~T}$ ) within the $0.22<\sin \theta / \lambda$ $<0.72$ range and $l$ index between -1 and 5 .

$F_{M}(\mathbf{Q}) \propto \sum_{j} \mu_{j \perp} f_{j}(\mathbf{Q}) e^{i \mathbf{Q} \cdot \mathbf{r}_{\mathbf{j}}}$, where $\mu_{j \perp}$ is the component of the $\mathbf{j}$-th magnetic moment perpendicular to the scattering vector $\mathbf{Q}$ and $f_{j}(\mathbf{Q})$ is the magnetic form factor of the $j$-th ion at position $r_{j}$ in the unit cell [27]. Since there are more $\mathrm{Bi}$ atoms in the unit cell, sum runs over all $j$ ions situated at 
position $r_{j}$ in the unit cell. However, all $\mathrm{Bi}$ atoms are assumed to have the same magnetic form factor $f(\mathbf{Q})$ and $\mu_{\perp}$ (component of the magnetic moment perpendicular to the scattering vector $\mathbf{Q}$ ), the index $j$ can be removed for these two terms and both factors put in front of summation. When unpolarized neutron beam is used, one records an intensity proportional to the sum of $\left|F_{M}(\mathbf{Q})\right|^{2}$ and the nuclear structure factor squared $\left|F_{N}(\mathbf{Q})\right|^{2} \propto\left|\sum_{j} b_{j} e^{i \mathbf{Q} \cdot \mathbf{r}_{\mathbf{j}}}\right|^{2}$. It is clear that if the magnetic moments are small, $\left|F_{M}(\mathbf{Q})\right|^{2}$ is negligible to $\left|F_{N}(\mathbf{Q})\right|^{2}$ and $F_{M}(\mathbf{Q})$ cannot be determined precisely. However, the sensitivity to the magnetic part can be greatly improved by the use of polarized neutron beam [23]. In such an experiment one then measures the intensities $I^{ \pm}(\mathbf{Q}) \propto\left|F_{N}(\mathbf{Q}) \pm F_{M}(\mathbf{Q})\right|^{2}$, where the + and - sign refer to up and down polarization directions of the incoming neutron beam. One then collects flipping ratios $R(\mathbf{Q})=I^{+}(\mathbf{Q}) / I^{-}(\mathbf{Q})$ and magnetic structure factors are determined with the knowledge of nuclear structure factors. These are normally determined in a separate diffraction experiment leading to the crystal structure parameters and other relevant parameters like the extinction of the crystal and thermal Debye-Waller factors. In our case, since the magnetic contribution is extremely small, the intensities recorded in the PND experiments represent very well the nuclear intensities. The magnetic signal modifies the experimental intensities by less than than $0.2 \%$. Therefore could be used in the first approximation for the crystal structure refinement. Indeed, the refined structural parameters neglecting the deviations of $0.2 \%$ caused by the magnetic contribution are in good agreement with the literature data. It appears that both, absorption and extinction effects can be neglected.

Although the largest signal detected in the experiment at $2 \mathrm{~K}$ for field of 6.2 Twas at a level of $5 \sigma$, only fifteen flipping ratios showed signal, defined as a difference from unity that is at least 1.5 times larger than associated statistical error documenting that the magnetic contribution is very small. Error bars obtained with field of $4.5 \mathrm{~T}$ were even larger. This manifests the necessity to use polarized neutrons. These reflections, together with the bulk magnetization inferred from the magnetic bulk experiment were included in further analysis. 


\section{Results}

\subsection{Magnetic bulk measurements}

In Fig. 1 we show the field dependence of the magnetization measured at $2 \mathrm{~K}$ in fields up to $14 \mathrm{~T}$. As it was expected, it is negative and shows almost a linear decrease with increasing field. There is no hysteresis between the sweep-up and sweep-down branches. The magnetization at $2 \mathrm{~K}$ and $6.2 \mathrm{~T}$ amounts $-0.0031 \mu_{B} / \mathrm{Bi}$ atom. At $4.5 \mathrm{~T}$ the magnetization amounts to $-0.0022 \mu_{B} / \mathrm{Bi}$ atom. These values were adopted in the evaluation of the neutron diffraction data.

However, one can observe on the magnetization curve also oscillations. In the right inset of Fig. 1 we show the very same magnetization after subtraction of a straight line fitted to the data in the whole field range as a function of inverse magnetic field. Clearly, an oscillatory behavior with a frequency of $1 / \mu_{0} H=0.15 \mathrm{~T}^{-1}$ is observed. This value is in very good agreement with the period found in the case of the Nernst effect [20] that reflects predominantly response of hole pockets. The temperature dependence of the magnetic susceptibility measured in various fields is nearly field independent. In the left inset of Fig. 1 we show the magnetic susceptibility measured at $7.5 \mathrm{~T}$ applied nearly along the $c$-axis. Except for temperatures below $\approx 70 \mathrm{~K}$, where deviations are seen, its temperature dependence is linear with temperature and thus, does not follow a Curie-Weiss law. This is in agreement with literature [3].

\subsection{Polarized neutron diffraction}

One way to treat the PND experimental data is the direct refinement of the measured flipping ratios. Let us assume for a moment that all the field induced magnetic moments are centered on the bismuth atomic sites. Then, all experimentally determined magnetic form factors should fall on a single smooth curve as a function of $\sin \theta / \lambda$ - bismuth magnetic form factor $f(\mathbf{Q})$ that have in general orbital $\left(\mu_{L}\right)$ and spin $\left(\mu_{S}\right)$ parts. However, as it is evident from Fig. 2, experimental points do not follow the expected smooth curve. Besides the issues connected with the sensitivity of our experiment, another possible reason of that might be that there is at least one more contribution to the field-induced magnetization that is not connected with the electron states centered at bismuth sites. The experimentally determined most significant magnetic structure factors are listed in They are listed in Table 1. 


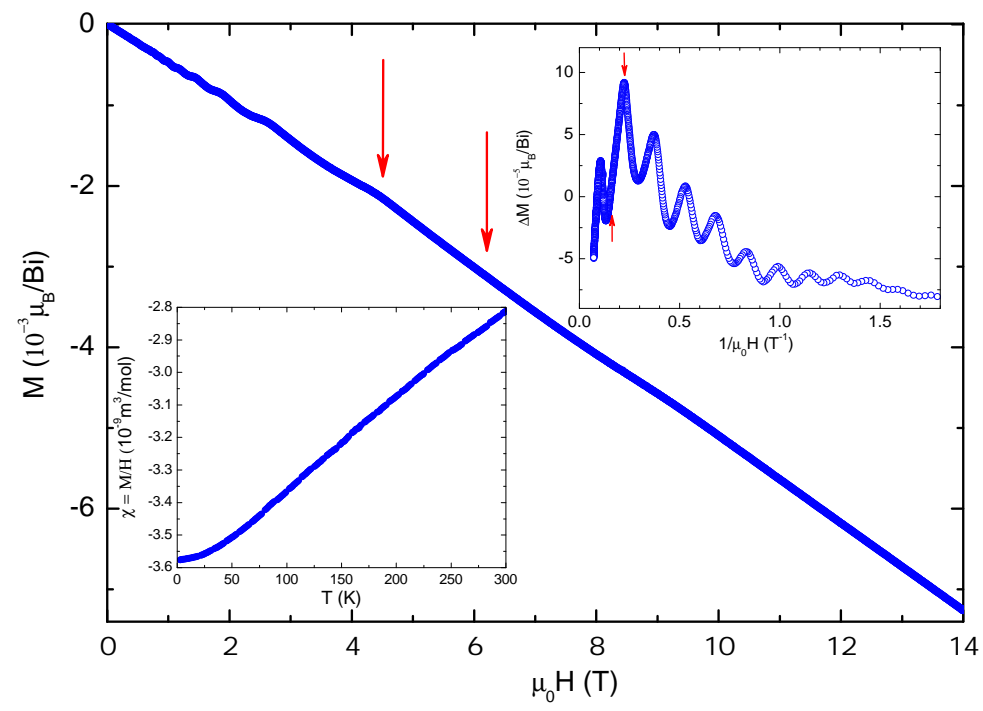

Figure 1: Field dependence of the magnetization measured at $2 \mathrm{~K}$ in fields up to $14 \mathrm{~T}$ applied along the $c$-axis on bismuth single crystal. The right inset shows the oscillatory character of the magnetic susceptibility after subtraction of a straight line. Red arrows indicate magnetic fields at which we have collected polarized neutron data. The left inset shows the temperature dependence of the magnetic susceptibility measured at $7.5 \mathrm{~T}$ applied along the same direction.

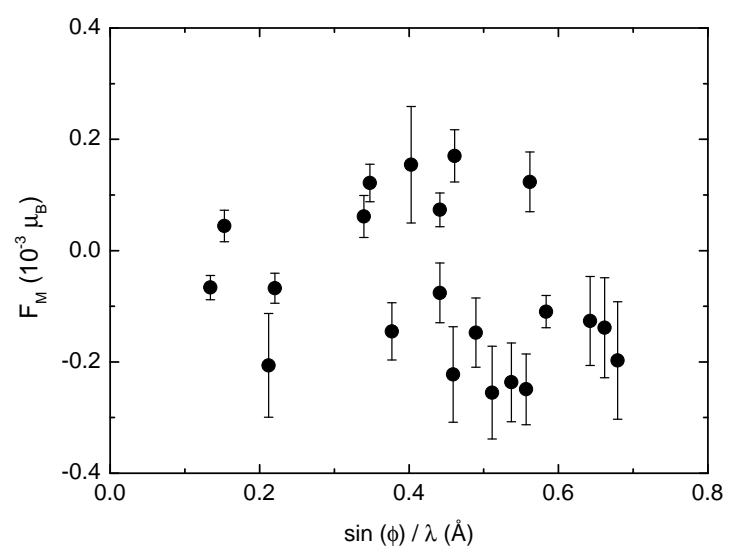

Figure 2: (Color online) Magnetic structure factors of bismuth single crystal measured at $2 \mathrm{~K}$ in field of $6.2 \mathrm{~T}$ applied along the trigonal axis as a function of $\sin (\theta) / \lambda$. 


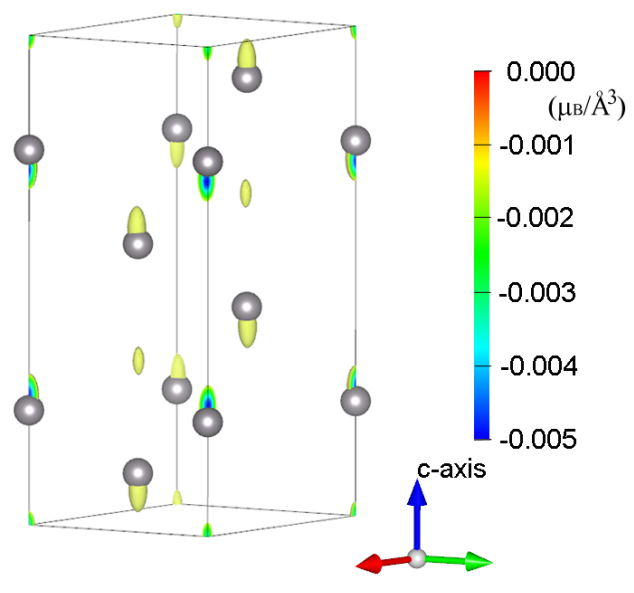

Figure 3: (Color online) The magnetization density of bismuth obtained from the maximum entropy reconstruction of data obtained at $2 \mathrm{~K}$ with field of $6.2 \mathrm{~T}$ applied nearly along the $c$-axis. Bi atoms are shown as dark spheres. The color scale represents the density. The isosurface value is set to $-1.5 \cdot 10^{-3} \mu_{B} / \AA$.

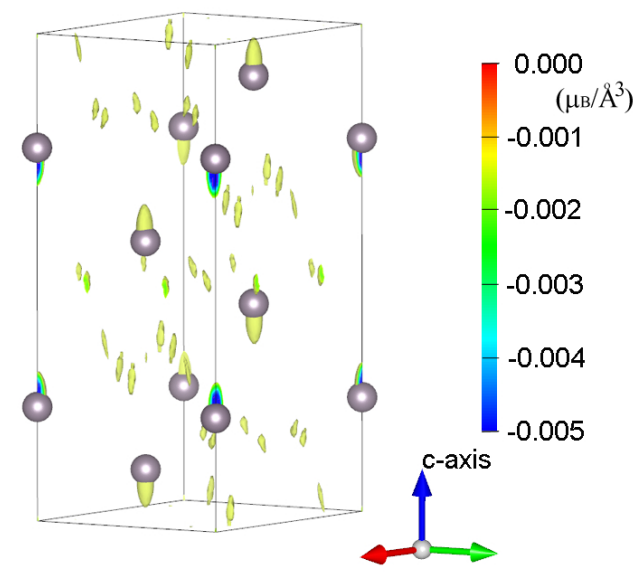

Figure 4: (Color online) The magnetization density of bismuth obtained from the maximum entropy reconstruction of data obtained at $2 \mathrm{~K}$ with field of $4.5 \mathrm{~T}$ applied nearly along the $c$-axis. Bi atoms are shown as dark spheres. The color scale represents the density. The isosurface value is set to $-1.5 \cdot 10^{-3} \mu_{B} / \AA$. 


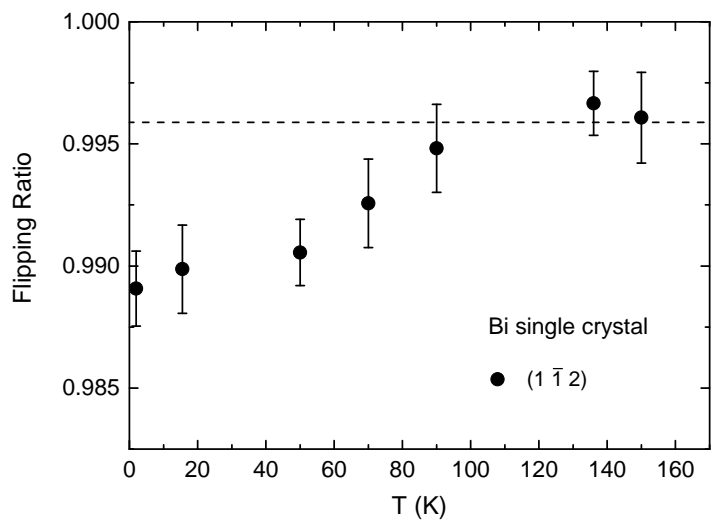

Figure 5: Temperature dependence of the flipping ratio measured on the bismuth single crystal (11̄2) reflection. The dashed line denotes high-temperature flipping-ratio value.

Another powerful and illustrative treatment of the data is the method of maximum entropy (MAXENT) [28]. This technique gives the most probable magnetization distribution map compatible with the measured structure factors and their experimental uncertainties. It does not need any a priori assumptions concerning the unmeasured data and no detailed atomic model is required. Merely the space group, the lattice constants and the flipping ratios together with the corresponding measured nuclear structure factors are needed. For the MAXENT reconstruction we have used Fortran based computer code PRIMA [29] and for visualization computer code VESTA [30]. The unit cell of bismuth was divided into $64 \times 64 \times 128=524288$ cells, in which the magnetization is assumed to be constant. The reconstruction was started from a flat magnetization distribution with a total moment in the unit cell equal to the bulk magnetization measured experimentally.

The most important result, namely the density map determined from the data acquired at $6.2 \mathrm{~T}$, together with the position of $\mathrm{Bi}$ atoms is shown in Fig. 3. The reconstruction map shows clearly clouds of negative density at position $(00 \approx 0.265)$ (and symmetry-equivalent positions), in a close vicinity of bismuth atoms that are situated at $(00 \approx 0.237)$. In addition, less pronounced negative clouds are found at $\left(\begin{array}{lll}0 & 0 & 0\end{array}\right)$ between Bi atoms. All clouds are seemingly elongated in the $c$-axis direction which is due to a reduced experimental resolution along this direction. Integration around these positions lead to moment of $-1.5 \cdot 10^{-3} \mu_{B}$. This moment, which we 
associate with the field-induced bismuth magnetic moment, is to be compared with value $-3.1 \cdot 10^{-3} \mu_{B}$ from magnetization measurements (see the right arrow in Fig. 1). The discrepancy between both values suggests that an appreciable amount of magnetization originates from states connected with interstitial space.

In Fig. 4 we show the the MAXENT reconstruction map obtained from data collected at $2 \mathrm{~K}$ with field of $4.5 \mathrm{~T}$, i.e. at the maximum of the magnetization after subtraction of a linear field dependence (see the left inset of Fig. 1). At these conditions, the magnetization per $\mathrm{Bi}$ atom is $-2.2^{-1} 10^{-3}$ $\mu_{B}$. As in the case of the higher field, we do observe clear clouds of a negative magnetization at $(00 \approx 0.265)$. The integrated moment around this position leads to a value of $-0.9 \cdot 10^{-3} \mu_{B} / \mathrm{Bi}$.

We see, that in both cases is the local moment, determined from integration of the MAXENT density map around Bi atomic positions by more that factor of two lower than the expected moment determined from magnetization measurement. This discrepancy is due to negative magnetization at positions outside the $\mathrm{Bi}$ atoms.

\subsection{Temperature dependence}

By following the field and temperature developments of flipping ratios of various Bragg reflections it should be, in principle, possible to extract various contributions [24]. However, we have been able to follow merely two Bragg reflections. The temperature dependence of the flipping ratio measured on the (112) reflection is shown in Fig. 5. Clearly, below about $90 \mathrm{~K}$ one observes that the signal becomes stronger suggesting an existence of at least two different contributions. The temperature of $90 \mathrm{~K}$ is similar to temperature below which one observes deviations from the high temperature magnetic susceptibility dependence and appearance of oscillatory phenomena.

\section{Discussion and Conclusions}

Our PND experiment clearly shows negative magnetization clouds in a close vicinity of the $\mathrm{Bi}$ atomic positions with additional magnetization between them. This result suggests that electrons centered at bismuth atoms are responsible for the signal detected in our PND experiment only partially. Similar conclusion has been drawn for bismuth by Wilkinson [31] and by Wilkinson et al. [32] for graphite. The negative magnetization signal in our 
experiment has three-fold symmetry imposed by the crystal structure symmetry used in the analysis. On the other hand, experiments in strong magnetic fields $[12,18,20,33]$ indicate that the three-fold electron pocket valley degeneracy is lifted in fields inclined with respect to the trigonal axis leading to quantum phenomena. In our case, the deviation of the field direction from the $c$-axis was 3.5 degrees and it is evident that three-fold symmetry is in our experiment slightly disturbed. This puts our maximum entropy reconstruction (that assumed the paramagnetic space group $\mathrm{R} \overline{3} 2 / \mathrm{m}$ ) in question and might indicate that one would need to use in the analysis lower symmetry. Nevertheless, lowering it down to $\mathrm{P} \overline{1}$ does not lead to much better agreement between the observed and calculated magnetic structure factors (not shown). We therefore conclude that one can still use the full symmetry of space group $\mathrm{R} \overline{3} 2 / \mathrm{m}$.

Our MAXENT analysis points to different field-induced contributions. The main issue here is to separate different contributions originating from electrons and holes on one side and a bare magnetic susceptibility on the other by determining the magnetization distribution at the top and bottom of the oscillations at $4.5 \mathrm{~T}$ and $6.2 \mathrm{~T}$. It appears that the $\mathrm{Bi}$ magnetic moment is nearly linearly proportional to the applied magnetic field and the tiny oscillations visible on the magnetization curve do not lead a substantially different magnetization distributions. Perhaps, another way to solve the problem and to separate the pocket contribution from others sources would be to conduct PND experiment with magnetic field of $2.5 \mathrm{~T}$ applied along the bisectrix direction. In this configuration, all carriers in all three electron pockets should reach their lowest spin polarized Landau level [34], maximizing their contribution to the magnetic susceptibility.

Nevertheless, both MAXENT magnetization maps and the temperature dependence shown in Fig. 5 suggest that besides the field-induced $\mathrm{Bi}$ moments also other electronic states contribute significantly to the diamagnetic response of bismuth. In conclusion, we have performed combined study comprising magnetic bulk measurements and single crystal polarized neutron experiment with the aim to identify various contributions to the diamagnetic response of bismuth. We have found that at least two source exist. One, atomic-like can be associated with bismuth atoms and the other rather spread in the unit cell. Clear experimental identification of the contribution leading to oscillatory behavior of the magnetization (de Haas - van Alphen effect) due to crossing of Landau levels with the Fermi level could not be, due to a limited sensitivity of otherwise extremely sensitive polarized neutron 
diffraction, disclosed.

We acknowledge LLB for allocation of beamtime for performing the PND experiment.

[1] M. D. Simon, and A. K. Geim, Journal of Applied Physics 87, 6200 (2000)

[2] N. B. Brandt, M. V. Semerov and L. A. Falkovsky, J. Low Temp. Phys. 27, 75 (1977).

[3] S. Otake, M. Momiuchi, N. Matsuno, J. Phys. Soc. Jpn. 491824 (1980).

[4] D. Shoenberg, Phil. Trans. R. Soc. A 245, 1 (1952); 248, 1 (1955).

[5] H. Fukuyama and R. Kubo, J. Phys. Soc. Jpn. 28, 570 (1979).

[6] X. Gonze, J.-P. Michenaud and J.-P. Vigneron, Physica Scripta 37, 785 (1988).

[7] F. A. Buot and J. W. McClure, Phys. Rev. B 6, 4525 (1972).

[8] E. N. Adams, Phys. Rev. 89, 633 (1960).

[9] J. W. McClure and D. Shoenberg, J. Low. Temp. Phys. 22, 233 (1976).

[10] M. Koshino and T. Ando, Phys. Rev. B 81, 195431 (2010)

[11] Y. Liu and R. E. Allen, Phys. Rev. B 52, 1566 (1995).

[12] H. Yang, Benoît Fauqué, L. Malone, A. B. Antunes, Z. Zhu, C. Uher and K. Behnia, Nature Comm. 1, 47 (2010).

[13] P. B. Alers and R. T. Webber, Phys. Rev. 91, 1060 (1953).

[14] X. Du, S.-W. Tsai, D. L. Maslov and A. F. Hebard, Phys. Rev. Lett. 94, 166601 (2005).

[15] H. Fukuyama, Ann. Phys. 15, 520 (2006).

[16] Y. Fuseya, M. Ogata and H. Fukuyama, Phys. Rev. Lett. 102, 066601 (2009).

[17] S. Murakami, Phys. Rev. Lett. 97, 236805 (2006). 
[18] B. Fauqué, B. Vignolle, C. Proust, J.-P. Issi and K. Behnia, New J. Phys. 11, 113012 (2009).

[19] Yu. V. Sharlai and G. P. Mikitik, Phys. Rev. B 83, 085103 (2011).

[20] K. Behnia, M.-A. Méasson and Y. Kopelevich, Phys. Rev. Lett. 98, 166602 (2007).

[21] I. A. Lukyanchuk, A. A. Varlamov, and A. V. Kavokin, Phys. Rev. Lett. 107, 016601 (2011).

[22] K. Walther, Phys. Rev. Lett. 16, 642 (1966).

[23] R. Nathans, M. T., Pigott, and C. G. Shull, J. Phys. Chem. Solids 6, 38 (1958).

[24] C. G. Shull and R. P. Ferrier, Phys. Rev. Lett. 10, 295 (1963).

[25] C. Stassis, Phys. Rev. Lett. 24, 1415(1970).

[26] A. Gukasov, S. Rodrigues, J.-L. Meuriot, Th. Robillard, A. Sazonow, B. Gillon, A. Laverdunt, F. Prunes and F. Coneggo, Physics Procedia 42, $150(2013)$.

[27] J. Rossat-Mignod, in: Methods of Experimental Physics Vol.23C, eds Sköld K. and Price D. L. (Academic Press, Inc.,New York, Dordrecht, 1987), p. 1.

[28] J. Skilling and S. F. Gull, in: Maximum Entropy and Bayesian Methods in Inverse Problems, eds C. R. Smith and W. T. Grandy, Jr. (D. Reidel Publishing Comp., Dordrecht, 1985), p. 83.

[29] F. Izumi and R. A. Dilanian, in: Recent Research Development in Physics, Vol.3 Part II (Transworld Research Network Trivandrum 2002), p. 699.

[30] K. Momma and F. Izumi, J. Appl. Cryst. 41, 653 (2008).

[31] C. Wilkinson, PhDThesis, Massatchusetts Institutre of Technology (1979).

[32] C. Wilkinson, D. A. Keen, P. J. Brown and J. B. Forsyth, J. Phys. Condens. Matter 1, 3833 (1989). 
[33] L. Li, J. G. Checkelsky, Y. S. Hor, C. Uher, A. F. Hebard, R. J. Cava and N. P. Ong, Science. 321, 547 (2008).

[34] Z. Zhu, B. Fauqué, Y. Fuseya and K. Behnia Phys. Rev. B 84, 115137 (2011). 
Table 1: Observed largest magnetic structure factors $F_{M}$ of Bi single crystal for different $(h k l)$ reflections obtained at $2 \mathrm{~K}$ with field applied close to the trigonal axis. $\sigma$ denotes one standard deviation.

\begin{tabular}{cccccc}
\hline \hline $\sin \theta / \lambda$ & $h$ & $k$ & $l$ & $F_{M}\left(\mu_{B}\right)$ & $\sigma$ \\
\hline 0.13423 & 1 & 0 & 1 & -0.00007 & 0.00002 \\
0.15300 & 1 & 0 & -2 & 0.00004 & 0.00003 \\
0.21205 & 1 & 0 & 4 & -0.00021 & 0.00009 \\
0.2206 & 1 & 1 & 0 & -0.00007 & 0.00003 \\
0.33963 & 2 & 1 & 1 & 0.00006 & 0.00004 \\
0.34748 & 2 & 1 & -2 & 0.00012 & 0.00003 \\
0.37722 & 2 & 1 & 4 & -0.00015 & 0.00005 \\
0.4027 & 3 & 0 & 3 & 0.00015 & 0.00010 \\
0.44121 & 2 & 2 & 0 & 0.00007 & 0.00003 \\
0.45917 & 2 & 2 & 3 & -0.00022 & 0.00009 \\
0.46118 & 3 & 1 & -1 & 0.00017 & 0.00005 \\
0.48952 & 3 & 1 & -4 & -0.00015 & 0.00006 \\
0.51122 & 4 & 0 & 1 & -0.00026 & 0.00008 \\
0.53693 & 4 & 0 & 4 & -0.00024 & 0.00007 \\
0.55679 & 3 & 2 & 1 & -0.00025 & 0.00006 \\
0.56161 & 3 & 2 & -2 & 0.00012 & 0.00005 \\
0.58366 & 4 & 1 & 0 & -0.00011 & 0.00003 \\
0.64245 & 5 & 0 & 2 & -0.00013 & 0.00008 \\
0.66181 & 3 & 3 & 0 & -0.00014 & 0.00009 \\
0.67927 & 4 & 2 & 2 & -0.00020 & 0.00011 \\
\hline \hline
\end{tabular}

